cardiopulmonary bypass (26). While powders of nitrite salts are present on almost every laboratory bench in the world, and nitrite anion preparations are currently available for human infusion in cyanide antidote kits, further clinical work is needed to address the safety and efficacy of this agent for the treatment of specific human diseases. At the very least, perhaps we should avoid mouthwash and feel slightly less guilty about eating hot dogs at the ball park.

1. Pegg, R.B., and Shahidi, F. 2000. Nitrite Curing of Meat. Food \& Nutrition Press. Trumbull, Connecticut, USA. 268 pp.

2. Duncan, C., et al. 1997. Protection against oral and gastrointestinal diseases: importance of dietary nitrate intake, oral nitrate reduction and enterosalivary nitrate circulation. Comp. Biochem. Physiol. A Physiol. 118:939-948.

3. Björne, H., et al. 2004. Nitrite in saliva increases gastric mucosal blood flow and mucus thickness. J. Clin. Invest. 113:106-114. doi:10.1172/JCI200419019.

4. Dougall, H.T., Smith, L., Duncan, C., and Benjamin, N. 1995. The effect of amoxycillin on salivary nitrite concentrations: an important mechanism of adverse reactions? Br. J. Clin. Pharmacol. 39:460-462.

5. Tannenbaum, S.R., Weisman, M., and Fett, D. 1976. The effect of nitrate intake on nitrite formation in human saliva. Food Cosmet. Toxicol. 14:549-552.
6. Ishiwata, H., Boriboon, P., Harada, M., Tanimura, A., and Ishidata, M. 1975. Changes of nitrite and nitrate concentration in incubated human saliva. J. Food Hyg. Soc. Jpn. 16:93-98.

7. Benjamin, N., et al. 1994. Stomach NO synthesis. Nature. 368:502.

8. McKnight, G.M., et al. 1997. Chemical synthesis of nitric oxide in the stomach from dietary nitrate in humans. Gut. 40:211-214.

9. Lundberg, J.O.N., Weitzberg, E., Lundberg, J.M., and Alving, K. 1994. Intragastric nitric oxide production in humans: measurements in expelled air. Gut. 35:1543-1546.

10. Pique, J.M., Whittle, B.J., and Esplugues, J.V. 1989. The vasodilator role of endogenous nitric oxide in the rat gastric microcirculation. Eur. J. Pharmacol. 174:293-296.

11. Brown, J.F., Hanson, P.J., and Whittle, B.J.R. 1992 Nitric oxide donors increase mucus gel thickness in rat stomach. Eur. J. Pharmacol. 223:103-104.

12. Wallace, J.L., and Miller, M.J. 2000. Nitric oxide in mucosal defense: a little goes a long way. Gastroenterology. 119:512-520.

13. Zhang, Z., et al. 1997. Human xanthine oxidase converts nitrite ions into nitric oxide (NO). Biochem. Soc. Trans. 25:524S

14. Millar, T.M., et al. 1998. Xanthine oxidoreductase catalyses the reduction of nitrates and nitrite to nitric oxide under hypoxic conditions. FEBS Lett. 427:225-228.

15. Li, H., Samouilov, A., Liu, X., and Zweier, J.L. 2003 Characterization of the magnitude and kinetics of xanthine oxidase-catalyzed nitrate reduction: evaluation of its role in nitrite and nitric oxide generation in anoxic tissues. Biochemistry. 42:1150-1159.

16. Forman, D., Al-Dabbagh, S., and Doll, R. 1985 Nitrates, nitrites and gastric cancer in Great
Britain. Nature 313:620-625.

17. Pobel, D., Riboli, E., Cornee, J., Hemon, B., and Guyader, M. 1995. Nitrosamine, nitrate and nitrite in relation to gastric cancer: a case-control study in Marseille, France. Eur. J. Epidemiol. 11:67-73.

18. Gonzalez, C. A et al. 1994. Nutritional factors and gastric cancer in Spain. Am. J. Epidemiol. 139:466-473.

19. Gladwin, M.T., et al. 2000. Role of circulating nitrite and S-nitrosohemoglobin in the regulation of regional blood flow in humans. Proc. Natl. Acad. Sci. U. S. A. 97:11482-11487.

20. Rassaf, T., et al. 2003. NO adducts in mammalian red blood cells: too much or too little? Nat. Med 9:481-483.

21. Rodriguez, J., Maloney, R.E., Rassaf, T., Bryan, N.S. and Feelisch, M. 2003. Chemical nature of nitric oxide storage forms in rat vascular tissue. Proc. Natl. Acad. Sci. U. S. A. 100:336-341.

22. Kelm, M., Preik-Steinhoff, H., Preik, M., and Strauer, B.E. 1999. Serum nitrite sensitively reflects endothelial NO formation in human forearm vasculature: evidence for biochemical assessment of the endothelial L-arginine-NO pathway. Cardivasc. Res. 41:765-772.

23. Kleinbongard, P., et al. 2003. Plasma nitrite reflects constitutive nitric oxide synthase activity in mammals. Free Radic. Biol. Med. 35:790-796.

24. Cosby, K., et al. 2003. Nitrite reduction to nitric oxide by deoxyhemoglobin vasodilates the human circulation. Nat. Med. 9:1498-1505

25. Doyle, M.P., Pickering, R.A., DeWeert, T.M., Hoekstra, J.W., and Pater, D. 1981. Kinetics and mechanism of the oxidation of human deoxyhemoglobin by nitrites. J. Biol. Chem. 256:12393-12398.

26. Reiter, C.D., et al. 2002. Cell-free hemoglobin limits nitric oxide bioavailability in sickle-cell disease. Nat. Med. 8:1383-1389.

\section{New viruses shake old paradigms}

\section{Chen-Yu Wang and Bill Sugden}

McArdle Laboratory for Cancer Research, University of Wisconsin-Madison, Madison, Wisconsin, USA

Kaposi sarcoma-associated herpesvirus (KSHV) can establish latent infection in host cells. The latently infected cells can survive and proliferate with a few viral genes expressed. However, in some Kaposi sarcoma cells, KSHV undergoes a productive life cycle and causes cell lysis. A new study (see the related article beginning on page 124) demonstrates that, after KSHV infection or introduction of viral plasmids into host cells, viral DNA is rapidly lost. Lytic virus production with ensuing infections could balance the loss of the viral plasmids to maintain the virus in cancer cells.

J. Clin. Invest. 113:21-23 (2004). doi:10.1172/200420662.

\footnotetext{
Address correspondence to: Bill Sugden, McArdle Laboratory for Cancer Research, University of Wisconsin-Madison, 1400 University Avenue, Madison, Wisconsin 53706, USA. Phone: (608) 262-6697; Fax: (608) 262-2824;

E-mail: sugden@oncology.wisc.edu. Conflict of interest: The authors have declared that no conflict of interest exists. Nonstandard abbreviations used: Kaposi sarcoma-associated herpesvirus (KSHV); Epstein-Barr virus (EBV); Kaposi sarcoma (KS); latency-associated nuclear antigen 1 (LANA1); KSHV G protein-coupled receptor (vGPCR).
}

Tumor viruses cause at least $15 \%$ of human cancers. These oncogenic viruses have been identified by the retention of their genomes in precursors to tumor cells and in frank tumor cells. Two members of the herpesvirus family, Kaposi sarcoma-associated herpesvirus (KSHV) and Epstein-Barr virus (EBV), are human oncogenic viruses. Herpesviruses kill infected host cells during the production of progeny virus; the generation of virus would have to be avoided in order for infected cells to be sustained as tumors. KSHV and EBV cause several human lymphomas, and these tumor cells conform to our expectations: the tumor cells maintain the viral DNA extra-chromosomally, they express few viral genes, they rarely support virus production, and thus they are classified as being infected latently. However, KSHV-associated Kaposi sarcoma (KS), the cancer for which this virus is named, does not so obviously conform to our expectations. Instead, lytic replication of KSHV is usually found in some cells within KS lesions.

KS lesions contain both spindleshaped endothelial cells that are latently infected with KSHV as well as cells that support the production of progeny virus. In this issue of the JCI, Grundhoff and Ganem document a striking feature of infection with KSHV, which may provide part of the reason for the lytic replication KSHV undergoes in some cells of KS lesions (1). The authors exposed multiple human and murine cell lines and a primary human endothelial cell strain to KSHV and showed that cells are infect- 


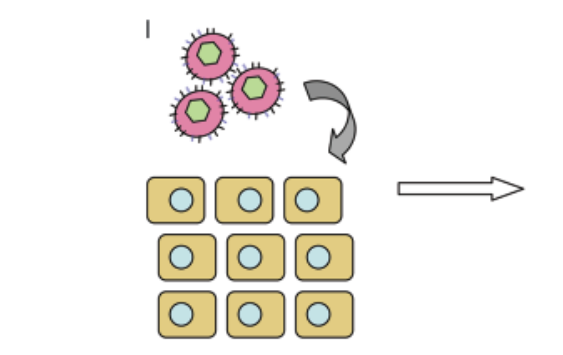

II
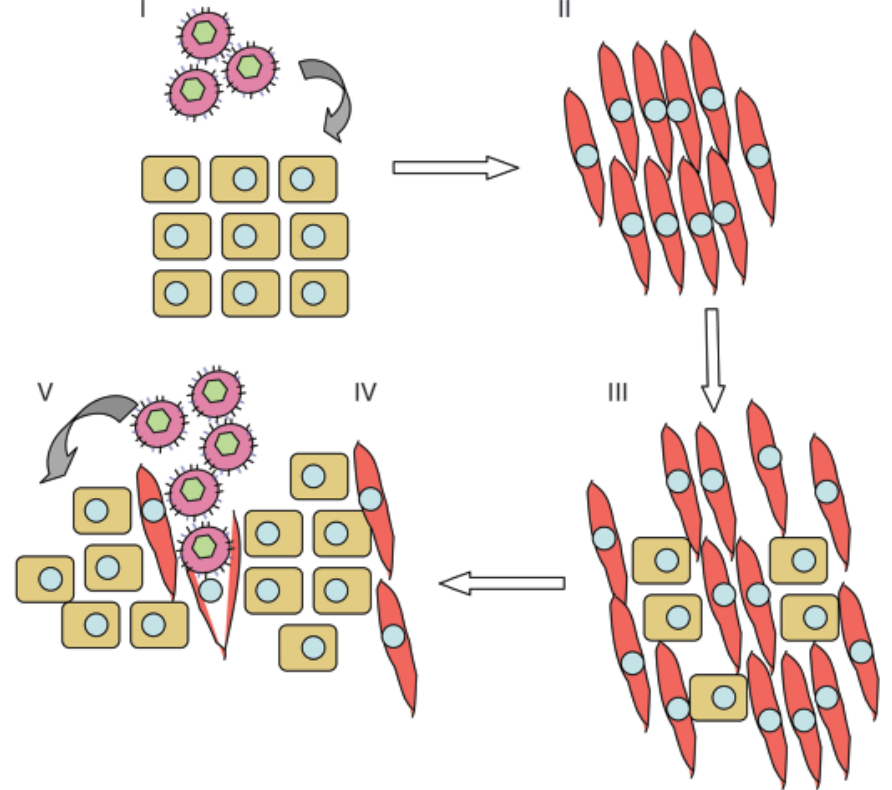

\section{Figure 1}

A model to explain one potential role for productive infections by KSHV in KS lesions. (I) KSHV (shown as an enveloped iscosahedral core) can infect cuboidal endothelial cells and (II) induce a spindle-shaped morphology in these cells. These infected spindle-shaped cells can proliferate, lose KSHV rapidly, and (III) revert to uninfected, cuboidal cells. (IV) The spindle-shaped cells also can support production of KSHV. (V) The released KSHV can infect more cells, inducing the change to the spindle shape and maintenance of the infected lesion.

ed via expression of latency-associated nuclear antigen 1 (known as LANA1), the viral protein that is required for viral DNA replication. They found that the infected, proliferating cells lost the virus within several days and up to 3 weeks after initial infection. The initially infected endothelial cells also rapidly lost their spindle shape. One cell line derived from KS and one fibroblast cell line maintained latent infections in approximately $10 \%$ of cells. The authors explored the mechanism of this general loss of the viral plasmid and infection by analyzing KSHV-infected cells. They also examined the fate of transfected plasmid replicons derived from KSHV, which contain the viral DNA replication origin (terminal repeat), with the expression of LANA1 in cis or in trans. Although these plasmids did replicate, they, like intact KSHV, were rapidly lost from most proliferating cells. These findings parallel those made with plasmid replicons derived from EBV (2), which are also initially rapidly lost from cells, although the plasmids themselves replicate. The traditional selection for plasmids that encode resistance to drugs has probably obscured this common, initial, rapid loss and explains our late recognition of this event.

Viruses such as KSHV and EBV, which maintain themselves extra-chromosomally in infected, proliferating cells, are lost from those cells unless they provide a selective growth or survival advantage. The work of Grundhoff and Ganem indicates that infection of multiple cell types generally leads to the loss of KSHV, showing that under these conditions KSHV fails to provide these proliferating cells with a selective advantage over uninfected cells (1). This observation may explain the difficulties researchers have had previously in documenting infection of cell lines with KSHV. In contrast, EBV can at least provide resting cells a selective advantage. Upon infecting primary $\mathrm{B}$ lymphocytes, EBV induces and maintains cell proliferation and is accordingly retained in the proliferating cells. The selective advantages that KSHV provides KS are now being assessed. Clearly, a fraction of two cell lines infected with KSHV retains the viral genome, indicating that in culture, KSHV does provide these cells with a selective advantage.

\section{A possible contribution of the lytic cycle to oncogenesis}

Grundhoff and Ganem propose that the rapid loss of KSHV from infected cells that they have observed in vitro likely occurs in endothelial cells in vivo and may be one reason for the frequent productive infection observed in spindle cells within KS lesions: new viruses would be required to recruit newly infected cells to the lesion (1). This proposition is consistent with the recent work of Ciufo et al. (3), who have found that KSHV infection of primary dermal microvascular endothelial cells yielded mixtures of latently infected spindle-shaped cells and small populations of productively infected cells (3). The virus released by the latter population was able to infect freshly added endothelial cells in order to maintain the mixed infected population (Figure 1). Other features of productive infection are also likely to contribute to KS. Viral genes thought not to be expressed during latent infection, based on analyses of KSHV-positive lymphoma cells, may be pivotal for the proliferation of KSHV-infected endothelial cells. KSHV G protein-coupled receptor (vGPCR), which is expressed early in productive infection, is one such viral gene. By itself, vGPCR can immortalize human primary endothelial cells and induce KS-like lesions in a mouse $(4,5)$.

A traditional hallmark of herpesviruses has been their latent infection, a relationship between the virus and host cell in which infected cells survive and the virus persists with little expression of its genes and without the production of progeny virus. The generality of this paradigm may be waning as the relationships between new herpesviruses and host cells are elucidated. Analysis of KSHV infection of endothelial cells has indicated that these cells may transition readily from latent to productive infection and that these transitions likely contribute to the pathogenesis of KS.

\footnotetext{
1. Grundhoff, A., and Ganem, D. 2004. Inefficient establishment of KSHV latency suggests an additional role for continued lytic replication in Kaposi sarcoma pathogenesis. J. Clin. Invest. 113:124-136. doi:10.1172/JCI200417803.

2. Leight, E., and Sugden, B. 2001. Establishment of
} 
an oriP replicon is dependent upon an infrequent, epigenetic event. Mol. Cell. Biol. 21:4149-4161.

3. Ciufo, D.M., et al. 2001. Spindle cell conversion by Kaposi's sarcoma-associated Herpesvirus: Formation of colonies and plaques with mixed lytic and latent gene expression in infected primary dermal microvascular endothelial cell cultures J. Virol. 75:5614-5626.

4. Bais, C., et al. 2003. Kaposi's sarcoma associated herpesvirus G protein-coupled receptor immortalizes human endothelial cells by activation of the VEGF receptor-2/KDR. Cancer
Cell. 3:131-143.

5. Montaner, S., et al. 2003. Endothelial infection with KSHV genes in vivo reveals that vGPCR initiates Kaposi's sarcomagenesis and can promote the tumorigenic potential of viral latent genes. Cancer Cell. 3:23-36.

\section{East meets West: an herbal tea finds a receptor}

\author{
Mitchell A. Lazar
}

Division of Endocrinology, Diabetes, and Metabolism, Departments of Medicine and Genetics, and the Penn Diabetes Center, University of Pennsylvania School of Medicine, Philadelphia, Pennsylvania, USA

Jaundice, which is caused by accumulation of bilirubin, is extremely common in newborn infants. Phototherapy is an effective treatment, but a drug therapy would also be desirable. A Chinese herbal remedy for jaundice called Yin Zhi Huang is now shown to activate a liver receptor that enhances the clearance of bilirubin (see the related article beginning on page 137). This discovery could lead to improved pharmaceutical treatments for neonatal jaundice.

J. Clin. Invest. 113:23-25 (2004). doi:10.1172/JCI200420661.
Advances in Western medicine have dramatically increased health and life expectancy (1). Nevertheless, over $\$ 4$ billion is spent per year on herbal remedies that are complementary or alternative to Western-style care (2). Prescription medicines are usually purified molecules whose biological target is established and whose efficacy is endorsed by regulatory agencies. By contrast, herbal medicines often contain many ingredients whose effects on biochemical pathways are unknown and whose efficacy is unproven in controlled studies. A report in the current issue of the JCI helps to bridge this gap. David Moore and colleagues (3) show the improvement of jaundice by a Chinese herbal tea called Yin Zhi Huang (YZH), "boiled down” to one component that regulates the activity of a nuclear receptor previously implicated in bilirubin clearance $(4,5)$. This discovery provides a mechanistic ration-

\footnotetext{
Address correspondence to: Mitchell A. Lazar, University of Pennsylvania School of Medicine, 611 Clinical Research Building, 415 Curie Boulevard, Philadelphia, Pennsylvania 19104-6149, USA. Phone: (215) 898-0198; Fax: (215) 898-5408;

E-mail: lazar@mail.med.upenn.edu.

Conflict of interest: The author has declared that no conflict of interest exists.

Nonstandard abbreviations used: Yin Zhi Huang (YZH); constitutive androstane receptor (CAR).
}

ale for pursuing an ingredient in $\mathrm{YZH}$ as a lead to improve upon the standard Western treatment for fetal jaundice.

\section{While there's tea, there's hope. -Sir Arthur Pinero}

Herbal remedies are often produced in the form of tea, that is, a decoction of dried plant leaves in boiling water. In today's Western world, teas serve as beverages for enjoyment as well as for herbal therapies. The former use has widespread acceptance, whereas the therapeutic use of tea is not espoused by mainstream Western medicine, largely because Western pharmacology has focused on purified chemical compounds, with defined mechanisms of action, whose effectiveness has been proven in controlled studies. By contrast, most teas used for medicinal purposes are admixtures of phytochemicals whose efficacy and biological target(s) are unproven by Western standards. This can threaten relationships between physicians guided by the Western medical literature and millions of their patients who opt to use teas preventively or therapeutically for cancer, inflammatory disease, and metabolic disease (6). At the same time, Eastern remedies may contain critical clues for disorders that have been refractory to Western medicine.

\section{Table 1}

Components of herbal remedies that target nuclear receptors

\begin{tabular}{|c|c|c|c|c|}
\hline Therapy & Indication & Compound & Receptor & Reference \\
\hline Rhei rhizoma ${ }^{\mathrm{A}}$ & Prostate cancer & Lindleyin & ER & 17 \\
\hline Ginseng & Stress & Ginsenoside-Rg1 & ER & 18 \\
\hline Grapeseed/red wine & Cardiovascular & Resveratrol & ER & 9 \\
\hline Scutellaria baicalensis ${ }^{\mathrm{B}}$ & Prostate cancer & Baicalein & AR & 19 \\
\hline Dioscorea villosa ${ }^{C}$ & Menopause & Diosgenin & PR & 20 \\
\hline Longmu Zhuanggu Chongji & Rickets & Vitamin D2 & VDR & 21 \\
\hline Xiao Chai Hu Tang & Leukemia & Retinoic acid & RAR & 22 \\
\hline Guggul tree resin & Lipid disorder & Guggulsterone & FXR & 23 \\
\hline Pseudolarix kaempferi & Fungal infection & Pseudolaric acid B & PPAR $\alpha$ & 24 \\
\hline Hypericum perforatum ${ }^{\mathrm{D}}$ & Depression ${ }^{\mathrm{E}}$ & Hyperforin & PXR & 25 \\
\hline Artemisia capillaris ${ }^{\mathrm{F}}$ & Jaundice & Dimethylesculetin & CAR & 15 \\
\hline Soy & Menopause & Genistein & $E R, A R, P R$ & 26 \\
\hline Labiatae $^{\mathrm{G}}$ & Lipid disorders & Isoprenoids & $\operatorname{PPAR} \alpha / \gamma$ & 27 \\
\hline
\end{tabular}

All examples are from traditional Chinese medicine except Guggul gum (Indian Ayurvedic), Dioscorea villosa (Mexican), and red wine. Note that not all examples could be listed, due to space constraints. Although several illustrative estrogen receptor compounds are shown, there are many environmental estrogens, which are reviewed elsewhere (27). ARhubarb. ${ }^{B}$ One of eight Chinese herbs in the herbal mixture PC-SPES (whose name is derived from PC, prostate cancer, and Latin spes, meaning "hope"): Isatis indigotica; Glycyrrhiza glabra and Glycyrrhiza uralensis (licorice); Panax pseudo-ginseng (ginseng); Ganoderma lucidum; Scutellaria baicalensis (skull cap); Dendranthema morifolium Tzvel (chrysanthemum); Rabdosia rubescens; and Serenoa repens (saw palmetto). 'Yam. DSt. John's wort. ENuclear receptor likely involved in toxicity rather than antidepression. FWormwood (component of Yin Zhi Huang and Yin Chin). GHerb family including basil, rosemary, oregano, and sage. ER, estrogen receptor; AR, androgen receptor; PR, progesterone receptor; VDR, vitamin D receptor; $R A R$, retinoic acid receptor; FXR, farnesyl X receptor (bile acid receptor); PXR, pregnane $X$ receptor; CAR, constitutive androstane receptor. 